TITLE:

\title{
Excited and ionized states of aniline: Symmetry adapted cluster configuration interaction theoretical study
}

\author{
$\operatorname{AUTHOR}(\mathrm{S})$ :
}

Honda, Y; Hada, M; Ehara, M; Nakatsuji, H

\section{CITATION:}

Honda, Y ... [et al]. Excited and ionized states of aniline: Symmetry adapted cluster configuration interaction theoretical study. JOURNAL OF CHEMICAL PHYSICS 2002, 117(5): 2045-2052

ISSUE DATE:

2002-08-01

URL:

http://hdl.handle.net/2433/50183

\section{RIGHT:}

Copyright 2002 American Institute of Physics. This article may be downloaded for personal use only. Any other use requires prior permission of the author and the American Institute of Physics. 


\title{
Excited and ionized states of aniline: Symmetry adapted cluster configuration interaction theoretical study
}

\author{
Yasushi Honda, Masahiko Hada, Masahiro Ehara, and Hiroshi Nakatsuji ${ }^{\text {a) }}$ \\ Department of Synthetic Chemistry and Biological Chemistry, Graduate School of Engineering, \\ Kyoto University, Sakyo-ku, Kyoto 606-8501, Japan
}

(Received 11 January 2002; accepted 30 April 2002)

\begin{abstract}
Singlet excited states and ionized states of aniline are studied by the symmetry adapted cluster/ configuration interaction method. Absorption bands of states that have mainly $\pi-\pi^{*}$ nature are assigned as ${ }^{1} A^{\prime \prime} \quad\left(\sim^{1} B_{2}\right),{ }^{1} A^{\prime} \quad\left(\sim^{1} A_{1}\right),{ }^{1} A^{\prime \prime} \quad\left(\sim^{1} B_{2}\right),{ }^{1} A^{\prime} \quad\left(\sim^{1} A_{1}\right),{ }^{1} A^{\prime \prime} \quad\left(\sim^{1} B_{2}\right)$ in increasing-energy order. An $s$-Rydberg state is predicted to lie between the first and second valence states, in agreement with recent experimental results. The lowest band has a charge-resonance character with a slight charge-transfer (CT) character (CT is defined as $\mathrm{NH}_{2} \rightarrow \mathrm{C}_{6} \mathrm{H}_{5}$ ); third and fifth valence bands have back-CT (BCT) nature, and second and fourth are local excitations within the benzene ring. The extent of $\mathrm{CT}$ of excited states depends on amino group conformation. In the planar form, CT characters of several states were altered; however, spectral shapes are very similar to that of the equilibrium form. On the other hand, amino group twisting altered both the spectrum and nature of excited states. Third and fourth lowest valence states exhibited strong CT character, while fifth to eighth states are of the strong BCT type, implying that the CT nature of excited states of aniline can be changed by amino group twisting. For ionized states, the lowest three states are assigned to ${ }^{2} A^{\prime}\left(\sim^{2} B_{1}\right),{ }^{2} A^{\prime \prime}\left(\sim^{2} A_{2}\right),{ }^{2} A^{\prime}\left(\sim^{2} B_{1}\right)$ in increasing-energy order, all being $\pi$-ionizations. The sixth one is also due to $\pi$-ionization $\left(\sim^{2} B_{1}\right)$ and the others are $\sigma$-ionizations. Ordering was the same as Koopmans' case. (C) 2002 American Institute of Physics.
\end{abstract}

[DOI: $10.1063 / 1.1487827]$

\section{INTRODUCTION}

It is well known that excited states of aromatic compounds exhibit large changes in molecular geometry and in acidity/basicity from their ground states. Aniline is one of the most important monosubstituted benzenes and is quite important for industry. Monosubstituted benzenes may be classified into two types, weak and strong substituent groups, from spectroscopic point of view. The weak one causes a spectral shift without any additional bands, whereas the strong one causes not only spectral shift, but also appearance of new bands owing to charge-transfers (CTs) between the substituent and the benzene ring. Aniline belongs to the latter group: the $\mathrm{NH}_{2}$ group is a strong substituent. This has caused some controversy in assignment of electronic spectra. ${ }^{1-6}$ For example, the second band was assigned to $\mathrm{CT}$ transition by Kimura $^{1,2}$ and Murrell, ${ }^{3}$ while Baba ${ }^{4}$ and Fischer-Hjalmars ${ }^{5}$ assigned it to a locally excited (LE) transition within the benzene ring; Suzuki and Fujii ${ }^{6}$ deemed it a mixed state having both characters.

Singlet electronic spectra of aniline have been observed in gas phase, ${ }^{1,2,7}$ in water, ${ }^{8}$ in perfluorohexane, ${ }^{9}$ and in crystals. ${ }^{7}$ Theoretical studies mostly have been used a semiempirical method: ${ }^{1-6}$ ab initio calculations are reported only by Bertinelli et al. in the single excitation configuration interaction (SECI) level with a minimal-class basis set; ${ }^{7}$ they are therefore very poor by today's theoretical standard. Moreover, all calculations were performed for a planar con-

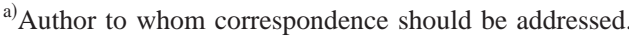

formation, despite the fact that the amino group is pyramidal in equilibrium geometry. This is a critical consideration for proper assignment, as shown in this paper.

Aniline ionization spectra were observed by Palmer et al. ${ }^{10}$ Kimura et al.,${ }^{11}$ and Kishimoto et al. ${ }^{12}$ Kishimoto et al. also reported Penning ionization electron spectroscopy (PIES) of aniline. ${ }^{12}$ Palmer et al. and Kimura et al. also performed Hartree-Fock calculations using double-zeta class basis sets and assigned their ionization peaks independently. ${ }^{10,11}$ Agreement of calculated energies with the experiment were rather poor however, and their assignments were different. Kishimoto et al. calculated the ionization potentials using the outer-valence Green's function (OVGF) method and gave assignments of the ionization peaks by comparing this calculation and PIES. ${ }^{12}$ However, these calculations may be inappropriate if effects of "shake-up" processes are not negligible.

For reliable theoretical assignments of excitation and ionization spectra, one should use highly accurate theory with an appropriately large basis set. Symmetry adapted cluster (SAC) (Ref. 13) and SAC-configuration interaction (CI) (Refs. 14, 15) methods are powerful methods to describe ground, excited, and ionized states of molecules which have been applied to various systems. ${ }^{16,17}$ This study examines the ground, singlet excited, and ionized states of aniline using the SAC/SAC-CI method. Calculated excitation and ionization energies and intensities agreed well with experimental results, indicating that our assignments are reasonable. 
Since we study vertical excitations and ionizations, we use the most stable geometry in which the amino group takes a pyramidal form. However, we also calculate two other conformations having planar and twisted amino groups. In the planar form, spectral shape is similar to the equilibrium one, but CT characters of several states are altered. On the other hand, the twisted form altered both the spectral shape and natures of the excited states. This indicates that aniline can have twisted intramolecular CT (TICT) states as in $N, N$-dimethylaminobenzonitrile (DMABN) ${ }^{18}$

\section{COMPUTATIONAL METHOD}

Ground, singlet excited, and doublet ionized states of aniline $\left(\mathrm{C}_{6} \mathrm{H}_{5} \mathrm{NH}_{2}\right)$ were calculated by the SAC/SAC-CI method. The basis set was Huzinaga-Dunning's $(9 s 5 p / 4 s) /[5 s 3 p / 3 s]$ with standard scaling factors ${ }^{19}$ plus Huzinaga's polarization functions ${ }^{20}$ ([1d] for $\mathrm{C}$ and $\mathrm{N},[1 p]$ for $\mathrm{H}$ ) and Dunning's diffuse anion basis ${ }^{21}$ ([2s2p] for $\mathrm{N}$ ) split with standard splitting factors. The total number of contracted basis functions was 190. Molecular geometry of aniline is due to electron diffraction experiments, ${ }^{22}$ while experimentally uncertain angles were optimized by MP2/ $[5 s 3 p / 3 s]+$ pol. $(d, p)+$ diff. $(s, p)$ calculations.

We used the SAC/SAC-CI SD- $R$ method in which single and double excitation operators are considered as linked operators. Hartree-Fock (HF) orbitals were used as reference orbitals. The active space consisted of 18 occupied orbitals and 158 unoccupied orbitals: only $1 s$ orbitals were frozen as core orbitals. All single excitation operators and some double excitation operators selected by perturbation selection procedure $^{23-25}$ were employed as linked operators. Selection thresholds were $5 \times 10^{-6}$ and $5 \times 10^{-7}$ for ground and excited states, respectively. For unlinked operators, $S_{2}^{2}, R_{1} S_{2}$, and $R_{2} S_{2}$ types were employed, where $S_{n}$ is a symmetryadapted $n$-electron excitation operator for SAC calculations and $R_{n}$ is that for SAC-CI calculations. Selection thresholds for unlinked terms were $\tau_{g}=0.005$ ( 0.002 for ionized states), $\tau=0.0005$ ( 0.001 for ionized states), and $\tau_{s}=\tau_{d}=0.05$; details of these thresholds are described elsewhere. ${ }^{25,26}$ All calculations were performed using the local version of the SAC-CI program ${ }^{26}$ combined with the GAUSSIAN 98 program package. $^{27}$

\section{SINGLET EXCITED STATES OF ANILINE}

\section{A. Hartree-Fock MOs}

Energies and natures of some HF orbitals of aniline are listed in Table I. Figure 1 shows the shapes of important orbitals. Superscripts + and - stand for symmetric and antisymmetric characters for the mirror plane. The $z$-axis is perpendicular to the mirror plane and the $x$-axis is perpendicular to the benzene ring (Fig. 1). The $\pi$ orbitals are $15 a^{\prime}$ (\#23; $\left.b_{1}\right), 9 a^{\prime \prime}\left(\# 24 ; \sim a_{2}\right), 16 a^{\prime}\left(\# 25 ; \sim b_{1}\right), 11 a^{\prime \prime}$ (\#30; $\sim a_{2}$ ), and $20 a^{\prime}\left(\# 31 ; \sim b_{1}\right)$. The \#24 and \#25 MOs correspond to degenerate $\pi$ MOs of benzene; \#30 and \#31 correspond to degenerate $\pi^{*}$ MOs. Although \#23 MO has a $\pi$ character, electron density is localized on the $\mathrm{N}$ atom and this orbital is assigned to $n$ nature. Other unoccupied MOs in this energy region have diffuse Rydberg natures.
TABLE I. Orbital energies and natures of some HF orbitals of aniline.

\begin{tabular}{|c|c|c|c|}
\hline $\begin{array}{c}\text { MO } \\
\text { number }^{\mathrm{a}}\end{array}$ & Symmetry $^{\mathrm{b}}$ & $\begin{array}{c}\text { Orbital } \\
\text { energy }(\mathrm{eV})\end{array}$ & Nature $^{c}$ \\
\hline \multicolumn{4}{|c|}{ Occupied orbitals } \\
\hline 14 & $10 a^{\prime}$ & -19.05 & $\sigma^{+}$ \\
\hline 15 & $5 a^{\prime \prime}$ & -18.44 & $\sigma^{-}$ \\
\hline 16 & $11 a^{\prime}$ & -17.71 & $\sigma^{+}$ \\
\hline 17 & $12 a^{\prime}$ & -16.19 & $\sigma^{+}$ \\
\hline 18 & $6 a^{\prime \prime}$ & -16.05 & $\sigma^{-}$ \\
\hline 19 & $7 a^{\prime \prime}$ & -15.74 & $\sigma^{-}$ \\
\hline 20 & $13 a^{\prime}$ & -14.48 & $\pi^{+}$ \\
\hline 21 & $14 a^{\prime}$ & -13.72 & $\sigma^{+}$ \\
\hline 22 & $8 a^{\prime \prime}$ & -13.26 & $\sigma^{-}$ \\
\hline 23 & $15 a^{\prime}$ & -12.18 & $\pi^{+}(n)$ \\
\hline 24 & $9 a^{\prime \prime}$ & -9.13 & $\pi^{-}$ \\
\hline 25 & $16 a^{\prime}$ & -8.07 & $\pi^{+}(\mathrm{HOMO})$ \\
\hline \multicolumn{4}{|c|}{ Unoccupied orbitals } \\
\hline 26 & $17 a^{\prime}$ & 1.55 & diff.( $s)$ (LUMO) \\
\hline 27 & $10 a^{\prime \prime}$ & 2.27 & $\operatorname{diff} .\left(p_{z}\right)$ \\
\hline 28 & $18 a^{\prime}$ & 2.49 & $\operatorname{diff.N}\left(p_{x}\right)$ \\
\hline 29 & $19 a^{\prime}$ & 3.04 & $\operatorname{diff} .\left(s, p_{y}\right)$ \\
\hline 30 & $11 a^{\prime \prime}$ & 3.44 & $\pi^{-*}$ \\
\hline 31 & $20 a^{\prime}$ & 4.04 & $\pi^{+*}$ \\
\hline 32 & $21 a^{\prime}$ & 7.14 & $\sigma^{+*}$, diff. $(s)$ \\
\hline
\end{tabular}

${ }^{a}$ Numbering starts from the lowest energy orbital including core orbitals. ${ }^{\mathrm{b}}$ See Fig. 1 and the text for the definition of molecular axes.

${ }^{\mathrm{c}}$ Superscripts,+- stand for symmetry with respect to the mirror plane; "diff" stands for a diffuse (Rydberg) nature.

\section{B. Singlet excited states for equilibrium conformation}

Table II shows energies and natures of singlet excited states of aniline calculated by the SAC/SAC-CI method together with experimental values. ${ }^{1,2}$ Figure 2 shows a comparison of the calculated spectrum with the experimental one. The SAC-CI theoretical spectrum is in good accordance

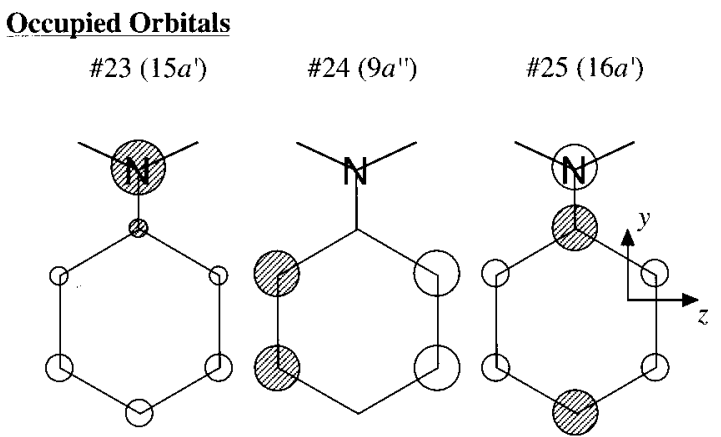

Unoccupied Orbitals

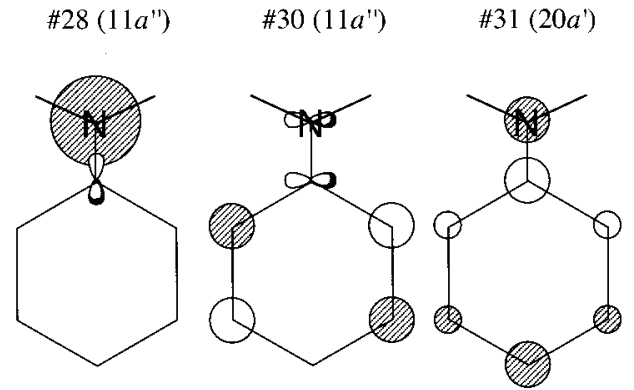

FIG. 1. Some important Hartree-Fock molecular orbitals of aniline. Molecular axes are shown in the figure. Circles on each atom stand for $\pi$-electron lobes. 
TABLE II. Excitation energies and natures of singlet excited states of aniline.

\begin{tabular}{|c|c|c|c|c|c|c|c|}
\hline \multicolumn{6}{|c|}{ SAC-CI } & \multicolumn{2}{|c|}{ Experiment $^{\mathrm{a}}$} \\
\hline State & Main configurations $(|C|>0.3)$ & Nature $^{b}$ & $\begin{array}{l}\text { Excitation } \\
\text { energy }(\mathrm{eV})\end{array}$ & $\begin{array}{l}\text { Oscillator } \\
\text { strength }\end{array}$ & $\begin{array}{l}\text { Second } \\
\text { moment (a.u.) }\end{array}$ & $\begin{array}{l}\text { Excitation } \\
\text { energy }(\mathrm{eV})\end{array}$ & $\begin{array}{l}\text { Oscillator } \\
\text { strength }\end{array}$ \\
\hline$X^{1} A^{\prime}$ & (ground state) & $\cdots$ & 0.00 & $\cdots$ & -90.13 & & \\
\hline $1^{1} A^{\prime \prime}$ & $0.83(25-30)+0.43(24-31)$ & $\pi^{+}-\pi^{-*}$ & 4.20 & 0.024 & -89.74 & 4.40 & 0.028 \\
\hline $2{ }^{1} A^{\prime}$ & $0.86(25-26)$ & $\pi^{+}$-Ryd. $(s)$ & 4.53 & 0.006 & -126.79 & & \\
\hline $3{ }^{1} A^{\prime}$ & $0.67(25-31)-0.53(25-28)-0.31(24-30)$ & $\pi^{+}-\pi^{+*}$ & 5.34 & 0.156 & -99.57 & 5.39 & 0.144 \\
\hline $2{ }^{1} A^{\prime \prime}$ & $0.90(25-27)$ & $\pi^{+}-\operatorname{Ryd} \cdot\left(p_{z}\right)$ & 5.57 & 0.001 & -133.07 & & \\
\hline $4{ }^{1} A^{\prime}$ & $0.87(25-29)$ & $\pi^{+}$-Ryd. $\left(s, p_{y}\right)$ & 6.06 & 0.012 & -129.06 & & \\
\hline $5{ }^{1} A^{\prime}$ & $0.69(25-28)+0.49(25-31)-0.36(24-30)$ & $\pi^{+}$-Ryd. $\left(p_{x}\right)$ & 6.26 & 0.001 & -122.52 & & \\
\hline $3{ }^{1} A^{\prime \prime}$ & $0.77(24-26)+0.33(24-29)$ & $\pi^{-}$-Ryd. $(s)$ & 6.39 & 0.078 & -120.57 & & \\
\hline $4{ }^{1} A^{\prime \prime}$ & $\begin{array}{l}0.59(24-31)-0.35(25-30)-0.35(24-29) \\
-0.33(23-30)-0.31(24-26)-0.30(24-28)\end{array}$ & $\pi^{-}-\pi^{+*}$ & 6.62 & 0.258 & -98.91 & 6.40 & 0.510 \\
\hline $6{ }^{1} A^{\prime}$ & $0.81(24-30)+0.42(25-31)$ & $\pi^{-}-\pi^{-*}$ & 6.87 & 0.722 & -93.80 & 6.88 & 0.570 \\
\hline $7{ }^{1} A^{\prime}$ & $0.94(24-27)$ & $\pi^{-}-\operatorname{Ryd} .\left(p_{z}\right)$ & 7.24 & 0.019 & -137.69 & & \\
\hline $5{ }^{1} A^{\prime \prime}$ & $0.80(24-29)-0.41(24-26)$ & $\pi^{-}$-Ryd. $\left(s, p_{y}\right)$ & 7.52 & 0.002 & -133.37 & & \\
\hline $6{ }^{1} A^{\prime \prime}$ & $0.76(24-28)-0.48(23-30)$ & $\pi^{-}$-Ryd. $\left(p_{x}\right)$ & 7.70 & 0.039 & -127.20 & & \\
\hline $8{ }^{1} A^{\prime}$ & $0.76(23-26)-0.33(25-26)$ & $\pi^{+}(n)$-Ryd. $(s)$ & 7.80 & 0.029 & -129.61 & & \\
\hline $9^{1} A^{\prime}$ & $0.95(22-30)$ & $\sigma^{-}-\pi^{-*}$ & 7.96 & 0.003 & -88.74 & & \\
\hline $7{ }^{1} A^{\prime \prime}$ & $0.61(23-30)+0.46(24-31)+0.40(24-28)$ & $\pi^{+}(n)-\pi^{-*}$ & 8.10 & 0.246 & -102.97 & 7.87 & $\sim 0.68$ \\
\hline $8{ }^{1} A^{\prime \prime}$ & $0.85(21-30)$ & $\sigma^{+}-\pi^{-*}$ & 8.37 & 0.028 & -89.37 & & \\
\hline $10{ }^{1} A^{\prime}$ & $0.80(25-32)+0.46(25-33)$ & $\pi^{+}-\sigma^{+*}$, Ryd. $(s)$ & 8.40 & 0.005 & -103.17 & & \\
\hline $9^{1} A^{\prime \prime}$ & $0.86(22-31)$ & $\sigma^{-}-\pi^{+*}$ & 8.41 & 0.001 & -89.91 & & \\
\hline
\end{tabular}

${ }^{a}$ References 1 and 2 .

${ }^{\mathrm{b}}$ See Table I footnote for notations.

with the experimental one. The five bands in the experimental spectrum are assigned to $1{ }^{1} A^{\prime \prime}\left(\sim^{1} B_{2}\right), 3{ }^{1} A^{\prime}\left(\sim^{1} A_{1}\right)$, $4{ }^{1} A^{\prime \prime} \quad\left(\sim{ }^{1} B_{2}\right), \quad 6{ }^{1} A^{\prime} \quad\left(\sim{ }^{1} A_{1}\right)$, and $7{ }^{1} A^{\prime \prime} \quad\left(\sim^{1} B_{2}\right)$ in increasing-energy order. The second moments of these states are as small as that of the ground state; they are mainly assigned to $\pi-\pi^{*}$ natures, although the $7^{1} A^{\prime \prime}$ state has an $n-\pi^{*}$ character. The $9^{1} A^{\prime}, 8{ }^{1} A^{\prime \prime}$, and $9^{1} A^{\prime \prime}$ states are also valence states and are assigned to $\sigma-\pi^{*}$ transitions.

Table III shows Mulliken charges and dipole moments of the ground and the five $\pi-\pi^{*}$ excited states of aniline calculated by the SAC/SAC-CI method. Experimentally observed dipole moments ${ }^{28-33}$ are also listed in the table. Calculated dipole moments of the ground and the first excited

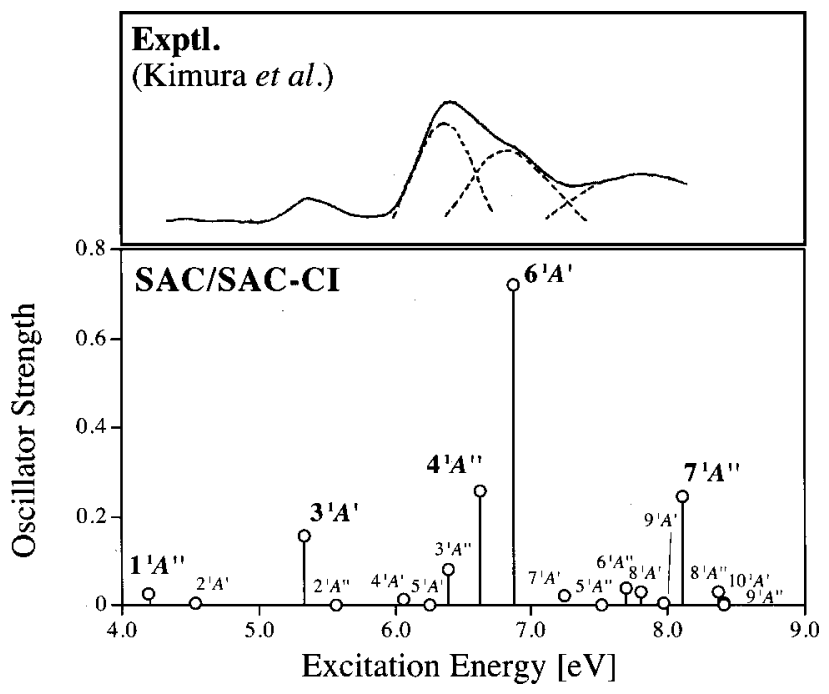

FIG. 2. Aniline electronic spectra by SAC-CI calculation and by gas phase experimentation (Refs. 1, 2). states agree well with experimental ones, though $\mu_{y}$ of the ground state is somewhat underestimated compared to the experimental value. Judging from configuration analysis, Mulliken charges, and dipole moments of these states, we assigned the five $\pi-\pi^{*}$ excited states to CR, LE, BCT, LE, and $\mathrm{BCT}$ in increasing-energy order, where LE stands for local excitation within the benzene ring, BCT for $\mathrm{C}_{6} \mathrm{H}_{5}$ $\rightarrow \mathrm{NH}_{2}$ back charge transfer, and $\mathrm{CR}$ for charge resonance resulting from mixing of $\mathrm{CT}$ and $\mathrm{BCT}$ characters (CT is $\mathrm{NH}_{2} \rightarrow \mathrm{C}_{6} \mathrm{H}_{5}$, whereas BCT is $\mathrm{C}_{6} \mathrm{H}_{5} \rightarrow \mathrm{NH}_{2}$ ). Although the first excited state $1^{1} A^{\prime \prime}$ is assigned to $\mathrm{CR}$, it has small CT character. The $1^{1} A^{\prime \prime}$ has the largest positive dipole moment in excited states below $8 \mathrm{eV}$. Our assignments on the CT characters are very different from those reported previously. ${ }^{1-6}$ It is probably attributed to that the BCT component has not been considered in the literature because of limitation of the virtual $\pi$ spaces.

Rydberg excited states of aniline ${ }^{7,9}$ and its derivatives ${ }^{34-36}$ have been reported. Recently, Ebata et al..$^{37}$ observed a new electronic state between the first and second $\pi-\pi^{*}$ excited states as a broad band in IR and $2+2$ ionization spectra. The state was found at $4.62 \mathrm{eV}$ above the ground state and at $0.38 \mathrm{eV}$ above the $1{ }^{1} A^{\prime \prime}$ excited state. In addition, they also observed a sharp signal at onset of the broad band in $2+2$ ionization spectra, which is assigned to the $0-0$ transition from the ground state to new excited states; the signal was not found in excitation spectra from $1{ }^{1} A^{\prime \prime}$. In our SAC-CI results, the $2{ }^{1} A^{\prime}$ state was calculated to be at $4.53 \mathrm{eV}$ and corresponds to ${ }^{1} B_{1}$ state in $C_{2 v}$ symmetry. This is consistent with the observation of Ebata et al. because excitation from $1{ }^{1} A^{\prime \prime}\left(\sim^{1} B_{2}\right)$ to $2{ }^{1} A^{\prime}\left(\sim^{1} B_{1}\right)$ is quasiforbidden while that from the ground state $\left(\sim^{1} A_{1}\right)$ is allowed. The $2{ }^{1} A^{\prime}$ state has a larger second moment than 
TABLE III. Properties of singlet excited states for equilibrium form.

\begin{tabular}{|c|c|c|c|c|c|c|c|c|c|}
\hline \multicolumn{7}{|c|}{ SAC-CI } & \multicolumn{3}{|c|}{ Experiment } \\
\hline \multirow[b]{2}{*}{ State } & \multirow{2}{*}{$\begin{array}{c}\text { Excitation } \\
\text { energy }(\mathrm{eV})\end{array}$} & \multicolumn{2}{|c|}{ Mulliken charge } & \multicolumn{2}{|c|}{ Dipole moment $(\mathrm{D})^{\mathrm{a}}$} & \multirow{2}{*}{$\begin{array}{c}\mathrm{CT} \\
\text { character }^{\mathrm{b}}\end{array}$} & \multirow{2}{*}{$\begin{array}{c}\text { Excitation } \\
\text { energy }(\mathrm{eV})\end{array}$} & \multicolumn{2}{|c|}{ Dipole moment $(\mathrm{D})^{\mathrm{a}}$} \\
\hline & & $\mathrm{C}_{6} \mathrm{H}_{5}$ & $\mathrm{NH}_{2}$ & $\mu_{y}$ & $|\boldsymbol{\mu}|$ & & & $\mu_{y}$ & $|\boldsymbol{\mu}|$ \\
\hline$X^{1} A^{\prime}$ & 0.00 & +0.05 & -0.05 & 0.84 & 1.49 & $\ldots$ & & $1.13^{\mathrm{d}}$ & $1.53,^{\mathrm{d}} 1.52^{\mathrm{e}}$ \\
\hline $1{ }^{1} A^{\prime \prime}$ & 4.20 & -0.05 & +0.05 & 2.12 & 2.39 & $\mathrm{CR}(\mathrm{CT})$ & $4.40^{c}$ & $1.98,{ }^{\mathrm{f}} 2.45,^{\mathrm{g}} 2.80^{\mathrm{h}}$ & $2.38^{\mathrm{f}}$ \\
\hline $3{ }^{1} A^{\prime}$ & 5.34 & +0.06 & -0.06 & 0.66 & 3.15 & LE & $5.39^{c}$ & & \\
\hline $4{ }^{1} A^{\prime \prime}$ & 6.62 & +0.15 & -0.15 & -0.18 & 2.57 & $\mathrm{BCT}$ & $6.40^{\mathrm{c}}$ & & \\
\hline $6{ }^{1} A^{\prime}$ & 6.87 & +0.02 & -0.02 & 1.37 & 1.78 & LE & $6.88^{\mathrm{c}}$ & & \\
\hline $7{ }^{1} A^{\prime \prime}$ & 8.10 & +0.21 & -0.21 & -2.47 & 3.15 & BCT & $7.87^{\mathrm{c}}$ & & \\
\hline
\end{tabular}

${ }^{a} \mu_{y}$ and $|\boldsymbol{\mu}|$ are the $y$-axis component and absolute value of the dipole moment, respectively.

${ }^{b} \mathrm{LE}=$ local excitation within the benzene ring, $\mathrm{CR}=$ charge resonance, $\mathrm{CT}=$ charge transfer $\left(\mathrm{NH}_{2} \rightarrow \mathrm{C}_{6} \mathrm{H}_{5}\right), \mathrm{BCT}=$ back-CT $\left(\mathrm{C}_{6} \mathrm{H}_{5} \rightarrow \mathrm{NH}\right)_{2}$.

${ }^{\mathrm{c}}$ References 1 and 2.

${ }^{\mathrm{d}}$ Reference 28 .

${ }^{\mathrm{e}}$ Reference 29.

${ }^{\mathrm{f}}$ Reference 30.

${ }^{g}$ References 31 and 32

${ }^{\mathrm{h}}$ Reference 33.

valence states and exhibits a diffuse character. Moreover, the oscillator strength is 0.006 , which is the order of typical Rydberg states. The $2^{1} A^{\prime}$ state is therefore expected to be associated with $\pi$-Rydberg states. To investigate this state in more detail, we performed SAC-CI calculations with an enlarged basis set comprising the above basis set plus Rydberg $(3 s, 3 p)$ functions on every $\mathrm{C}$ atom and split Rydberg $(4 s, 4 p, 4 d)$ on the center of the benzene ring. According to configuration analysis in this calculation, the $2{ }^{1} A^{\prime}$ state is found to be a $\pi$-Rydberg $(s)$ excited state. This assignment is further supported by observations of Rydberg states of $N, N$-dimethylaniline and $N, N$-diethylaniline. ${ }^{7,9,34-36}$ They were reported to be at $\approx 4.5-4.7 \mathrm{eV}$ for both molecules.

\section{Singlet excited states for planar conformation}

In many of reported calculations of excited states of aniline, the molecule was assumed to be planar. ${ }^{1-7}$ However, dependence of electronic states of aniline on amino group conformation has not been studied except for the ground and the lowest excited states. We calculated singlet excited states and associated electronic spectrum of the planar aniline together with those of the equilibrium form. Geometry was determined by MP2 optimization with the same basis set used for the equilibrium form, but with constraint of $C_{2 v}$ symmetry. Also, computational conditions for the SAC-CI method were the same as the equilibrium form. The HF orbitals for the planar form were similar to those for the equilibrium form and are not drawn explicitly.

Figure 3 shows the SAC-CI electronic spectra for three conformations: the equilibrium form, the planar form, and the twisted form (Sec. III D). One should note that principal axes of conformations differ from each other. In our definition, $A^{\prime}$ and $A^{\prime \prime}, \pi-\pi^{*}$ states for the equilibrium form correspond to $A_{1}$ and $B_{2}$ in $C_{2 v}$ symmetry, respectively. The spectral feature for the planar form is very similar to that for the equilibrium form. All states of the planar form can be corresponded to those of the equilibrium form. Intense bands consist of the five $\pi-\pi^{*}$ states, $1{ }^{1} B_{2}, 2{ }^{1} A_{1}, 2{ }^{1} B_{2}$, $4{ }^{1} A_{1}$, and $4{ }^{1} B_{2}$, in increasing-energy order, which corre- spond to $1^{1} A^{\prime \prime}, 3{ }^{1} A^{\prime}, 4{ }^{1} A^{\prime \prime}, 6{ }^{1} A^{\prime}$, and $7{ }^{1} A^{\prime \prime}$. Thus, effects of amino group wagging on electronic structure do not seem to be very large.

However, CT characters and dipole moments of excited states are greatly effected by conformational variation. Table
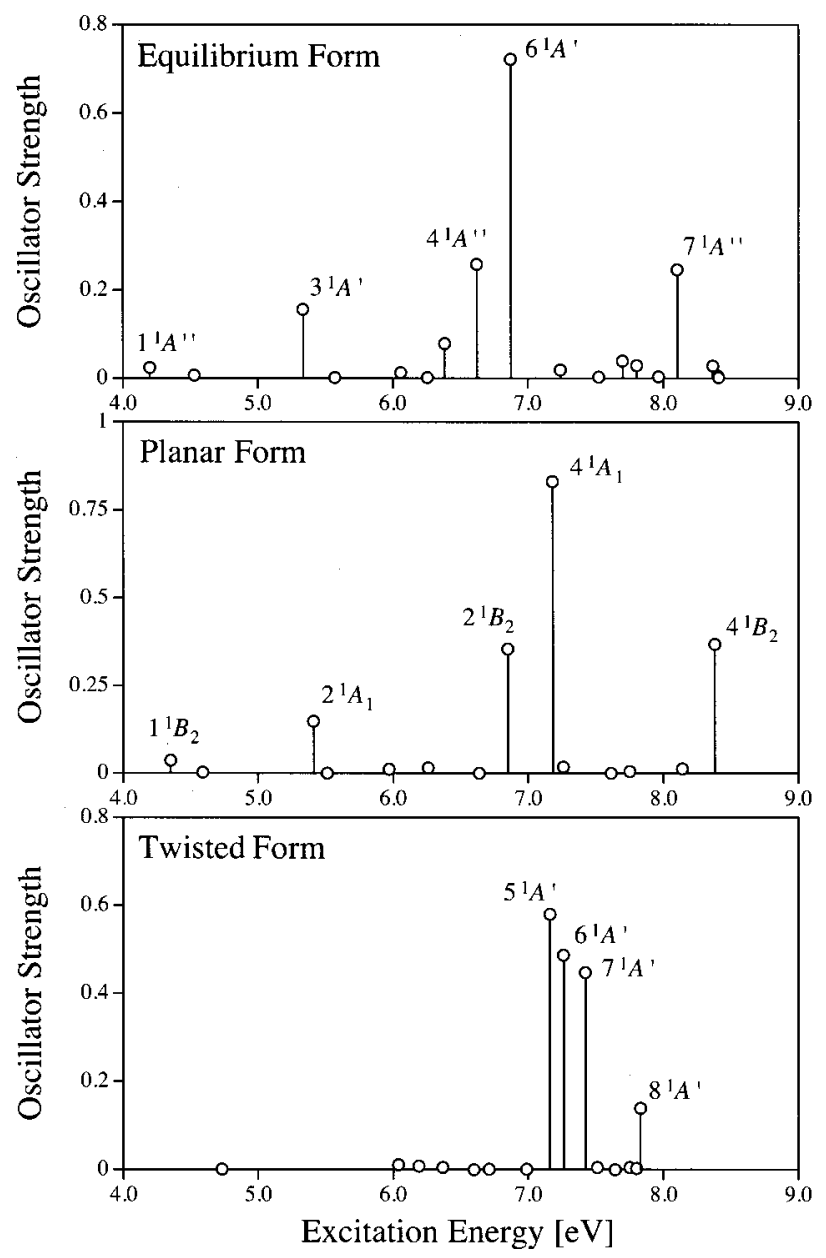

FIG. 3. Electronic spectra of aniline calculated by SAC-CI for three conformations: equilibrium form, planar form, and twisted form. 
TABLE IV. Excitation energies and properties of singlet excited states for planar form.

\begin{tabular}{|c|c|c|c|c|c|c|c|}
\hline \multicolumn{8}{|c|}{ SAC-CI } \\
\hline \multirow[b]{2}{*}{ State } & \multirow[b]{2}{*}{ Main configurations $(|C|>0.2)$} & \multirow{2}{*}{$\begin{array}{l}\text { Excitation } \\
\text { energy }(e \mathrm{~V})\end{array}$} & \multirow{2}{*}{$\begin{array}{l}\text { Oscillator } \\
\text { strength }\end{array}$} & \multicolumn{2}{|c|}{ Mulliken charge } & \multirow{2}{*}{$\begin{array}{c}\text { Dipole } \\
\text { moment }(\mathrm{D})^{\mathrm{a}}\end{array}$} & \multirow{2}{*}{$\begin{array}{c}\mathrm{CT} \\
\text { character }\end{array}$} \\
\hline & & & & $\mathrm{C}_{6} \mathrm{H}_{5}$ & $\mathrm{NH}_{2}$ & & \\
\hline$X^{1} A_{1}$ & (ground state) & 0.00 & $\cdots$ & +0.09 & -0.09 & 1.60 & $\cdots$ \\
\hline $1{ }^{1} B_{2}$ & $0.84(25-30)+0.41(24-31)$ & 4.35 & 0.037 & -0.04 & +0.04 & 3.05 & $\mathrm{CR}(\mathrm{CT})$ \\
\hline $2{ }^{1} A_{1}$ & $0.70(25-28)-0.57(25-31)+0.26(24-30)$ & 5.41 & 0.148 & +0.25 & -0.25 & -0.56 & $\mathrm{BCT}+\mathrm{LE}$ \\
\hline $2{ }^{1} B_{2}$ & $0.64(24-31)-0.47(24-28)-0.38(25-30)-0.35(23-30)$ & 6.85 & 0.353 & +0.11 & -0.11 & 1.78 & $\mathrm{CR}$ \\
\hline $4{ }^{1} A_{1}$ & $0.83(24-30)+0.41(25-31)$ & 7.18 & 0.830 & +0.04 & -0.04 & 2.47 & LE \\
\hline $4{ }^{1} B_{2}$ & $0.71(23-30)+0.48(24-31)+0.27(24-28)$ & 8.38 & 0.367 & +0.14 & -0.14 & -0.23 & $\mathrm{CR}(\mathrm{BCT})$ \\
\hline
\end{tabular}

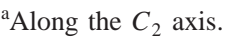

${ }^{b} \mathrm{LE}=$ local excitation within the benzene ring, $\mathrm{CR}=$ charge resonance, $\mathrm{CT}=$ charge transfer $\left(\mathrm{NH}_{2} \rightarrow \mathrm{C}_{6} \mathrm{H}_{5}\right), \mathrm{BCT}=$ back-CT $\left(\mathrm{C}_{6} \mathrm{H}_{5} \rightarrow \mathrm{NH} \mathrm{H}_{2}\right)$.

IV shows energies and properties of the ground and the five $\pi-\pi^{*}$ excited states for the planar form. From this information, we assign the five excited states to $\mathrm{CR}, \mathrm{BCT}+\mathrm{LE}, \mathrm{CR}$, $\mathrm{LE}$, and CR in increasing-energy order. For $1{ }^{1} B_{2}$, the CT character overcomes BCT; for $4{ }^{1} B_{2}$, BCT overcomes CT. The $2{ }^{1} A_{1}$ is assigned to a mixed state of BCT and LE characters. The CT characters for the planar form are, therefore, different from those for the equilibrium form. This implies that the assumption of planar conformation may provide incorrect aniline assignments, in particular for the CT characters. Wagging of the amino group from the planar form gives $\mathrm{BCT}, \mathrm{CT}, \mathrm{BCT}, \mathrm{BCT}$, and BCT characters for $1{ }^{1} B_{2}, 2{ }^{1} A_{1}$, $2{ }^{1} B_{2}, 4{ }^{1} A_{1}$, and $4{ }^{1} B_{2}$ states, respectively. The CT character of the ground state is unaffected by the wagging.

Change in CT character is explained in terms of mixing of $\pi$ and $\sigma$ natures. For example, the dipole moment of $1{ }^{1} B_{2}$ is larger than $1^{1} A^{\prime \prime}$ for the equilibrium form. This is explained by the following. The most dominant configuration of the lowest excited state is \#25-\#30 excitation for both forms. For the equilibrium form, \#30 MO slightly includes the $\sigma(\mathrm{N}-\mathrm{H})$ electron cloud, whereas such mixing is forbidden in the planar form and includes only the $\pi$ electron cloud on the benzene ring. Thus, CT contribution due to this configuration is more effective in the planar form. The $1{ }^{1} B_{2}$ state has the largest positive dipole moment in excited states, similarly to the equilibrium form.

\section{Singlet excited states for twisted conformation}

The twisted intramolecular CT (TICT) model is widely accepted to account for anomalous fluorescence of $\mathrm{N}, \mathrm{N}$-dimethylaminobenzonitrile (DMABN) in polar solvents; this phenomenon has been observed in various systems. ${ }^{18}$ For DMABN, the TICT state exhibits a strong CT character that is generated by twisting of the dimethylamino group. Although TICT states of aniline have been reported neither experimentally nor theoretically to our knowledge, it would be interesting to investigate twisted-aniline excited states. We calculated the singlet excited states and the electronic spectrum of aniline for the twisted amino group by $90^{\circ}$. Geometry was calculated by MP2 optimization with the same basis set as that for the equilibrium form, but with a constraint that the benzene ring is the mirror plane of $C_{s}$ symmetry. Computational conditions for the SAC-CI method were also identical to the equilibrium form.
The SAC-CI electronic spectrum for the twisted form is shown in Fig. 3 and compared with those of other conformations. Both spectral shape and nature of the excited states were largely altered by twisting; also, the spectrum is rather similar to that of benzene. No bands with large intensities were calculated up to $7 \mathrm{eV}$. Four strong bands observed at $\approx 7-8 \mathrm{eV}$ correspond to $5^{1} A^{\prime}, 6{ }^{1} A^{\prime}, 7{ }^{1} A^{\prime}$, and $8{ }^{1} A^{\prime}$ in increasing-energy order. They are assigned to mixed $\pi-\pi^{*}$, $\pi$-diff.N $\left(p_{z}\right)$ states. This is explained in terms of the change in the HF MOs, which are shown in Table V and Fig. 4. Note that the principal axis for the twisted form is different from that for the equilibrium form even though they have the same point group, $C_{s}$ : the axis perpendicular to the benzene ring is the $x$-axis for the latter, whereas it is the $z$-axis for the former. Therefore, all $\pi-\pi^{*}$ excited states belong to $A^{\prime}$ symmetry. The MOs also change greatly by twisting. The \#24 and \#25 MOs of the twisted aniline correspond to degenerate $\pi$ MOs of benzene, and \#30 and \#31 correspond to degenerate $\pi^{*}$ MOs of benzene, respectively, similarly to the equilibrium form. However, these MOs are much more localized at the benzene ring for the twisted form than the equilibrium form. Therefore, two quasiforbidden $\pi-\pi^{*}$ states of the twisted aniline were calculated at 4.73 and 6.19

TABLE V. Orbital energies and natures of important HF orbitals of twisted aniline.

\begin{tabular}{cccl}
\hline \hline $\begin{array}{c}\text { MO } \\
\text { number }\end{array}$ & Symmetry $^{\mathrm{b}}$ & $\begin{array}{c}\text { Orbital } \\
\text { energy (eV) }\end{array}$ & \multicolumn{1}{c}{ Nature $^{\mathrm{c}}$} \\
\hline \multicolumn{5}{c}{ Occupied orbitals } \\
23 & $21 a^{\prime}$ & -11.26 & $n$ \\
24 & $3 a^{\prime \prime}$ & -9.11 & $\pi^{-}$ \\
25 & $4 a^{\prime \prime}$ & -8.93 & $\pi^{+}(\mathrm{HOMO})$ \\
& & & \\
26 & $22 a^{\prime}$ & 1.66 & $\operatorname{diff.N}(s)(\mathrm{LUMO})$ \\
27 & $5 a^{\prime \prime}$ & 2.32 & $\operatorname{diff.N}\left(p_{z}\right), \sigma^{*}(\mathrm{~N}-\mathrm{H})$ \\
& $\cdots$ & & \\
30 & $6 a^{\prime \prime}$ & 3.36 & $\pi^{-*}$ \\
31 & $7 a^{\prime \prime}$ & 3.62 & $\pi^{+*}$ \\
\hline
\end{tabular}

${ }^{a}$ Numbering starts from the lowest energy orbital including core orbitals. ${ }^{\mathrm{b}}$ Definition of symmetries is different from the equilibrium form. The $a^{\prime}$ and $a^{\prime \prime}$ corrrespond to symmetric and antisymmetric characters with respect to the benzene plane, respectively.

${ }^{\mathrm{c}}$ Superscripts,+- stand for symmetry with respect to the $y z$ plane, which is the mirror plane for the equilibrium form. See Table I footnote for notation. 


\section{Occupied Orbitals}

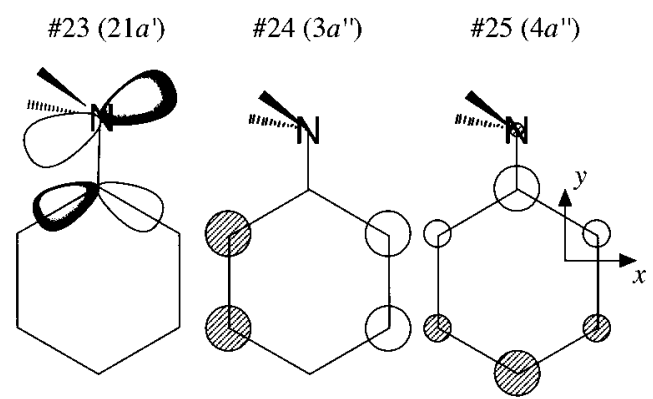

Unoccupied Orbitals

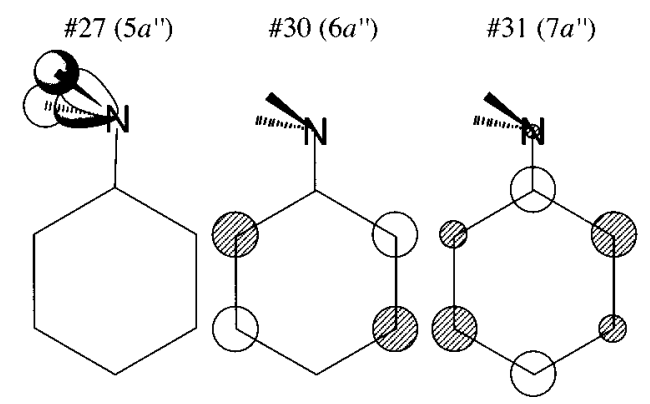

FIG. 4. Some important Hartree-Fock molecular orbitals of twisted aniline. Molecular axes are shown in the figure. Circles on each atom stand for $\pi$-electron lobes.

$\mathrm{eV}$, which are similar to ${ }^{1} B_{2 u}$ and ${ }^{1} B_{1 u}$ states of benzene [experimental values, $4.90 \mathrm{eV}$ (Ref. 38) and $6.20 \mathrm{eV}$ (Refs. $38,39)$, respectively]. The four $\pi-\pi^{*}$ states at $7-8 \mathrm{eV}$ correspond to the ${ }^{1} E_{1 u}$ states of benzene [experimental value, $6.94 \mathrm{eV}$ (Ref. 38)]. Splitting causes a breakdown of spatial degeneracy and different mixing between the $\pi-\pi^{*}$ and $\pi-$ diff.N $\left(p_{z}\right)$ excitations. The diff.N $\left(p_{z}\right)$, mainly \#27 MO, is a mixture of $\# 28$ diff. $\mathrm{N}\left(p_{x}\right) \mathrm{MO}$ and $\sigma^{*}(\mathrm{~N}-\mathrm{H}) \mathrm{MO}$ of the equilibrium form. Further, two quasiforbidden $n-\pi^{*}$ states were calculated at 6.60 and $6.99 \mathrm{eV}$ for the twisted form. They are excitations from \#23 MO, which is an $n$ MO caused by twisting of the \#23 $\pi$ MO for the equilibrium form.

Table VI shows details of energies, natures, and properties of the ground and valence excited states for the twisted form. Obviously, the third and fourth lowest valence excited states $\left(2{ }^{1} A^{\prime \prime}\right.$ and $\left.4{ }^{1} A^{\prime \prime} ; n-\pi^{*}\right)$ exhibited strong CT $\left(\mathrm{NH}_{2}\right.$ $\left.\rightarrow \mathrm{C}_{6} \mathrm{H}_{5}\right)$ characters, while the fifth to eighth states $\left[5^{1} A^{\prime}\right.$, $6{ }^{1} A^{\prime}, 7{ }^{1} A^{\prime}$, and $8{ }^{1} A^{\prime} ; \pi$-diff.N $\left.\left(p_{z}\right), \pi-\pi^{*}\right]$ showed strong BCT $\left(\mathrm{C}_{6} \mathrm{H}_{5} \rightarrow \mathrm{NH}_{2}\right)$ natures. Dipole moments of these excited states are much larger than those of any excited states of the equilibrium form. This indicates that aniline can have twisted intramolecular CT (TICT) states, similarly to $N, N$-dimethylaminobenzonitrile (DMABN) ${ }^{18}$ if the amino group is twisted. In contrast, dipole moments and Mulliken charges of first and second states $\left(2{ }^{1} A^{\prime}\right.$ and $\left.4{ }^{1} A^{\prime} ; \pi-\pi^{*}\right)$ are unchanged from the ground state and are assigned to LE states. This is the largest difference between aniline and DMABN. It is interesting that there exist strong BCT states as well as CT states in the twisted form. Introducing electron-donative groups to the benzene ring could promote this effect in BCT states, which is a challenging subject for future study.

\section{IONIZED STATES OF ANILINE}

Finally, we performed calculations and assignments of ionized states of aniline up to $20 \mathrm{eV}$. For ionized states, we performed perturbation selection of linked operators using not only singly-excited reference configurations, but also doubly excited reference configurations; we confirmed that both single- and SD-reference calculations provided similar results.

The SAC-CI results are shown in Table VII, in which Koopmans' result and experimental ${ }^{10-12}$ and other theoretical values $^{12}$ are also listed. Figure 5 shows the present theoretical ionization spectrum compared with experimental spectra reported by Kimura et al. ${ }^{11}$ and Palmer et al. ${ }^{10}$ Band intensities were calculated by monopole approximation. ${ }^{40}$ The calculated spectrum agrees well with experimental ones, inferring that these ionization band assignments are reliable. All bands up to $20 \mathrm{eV}$ are mainly due to one-electron ionization processes. Thus, the effect of the "shake-up" processes on spectra would be small.

For aniline, Koopmans theorem gives correct ionization band ordering, though ionization energies are rather poor. However, we note that Koopmans theorem with small basis

TABLE VI. Excitation energies, natures, and properties of singlet excited states for twisted form.

\begin{tabular}{|c|c|c|c|c|c|c|c|c|c|}
\hline \multicolumn{10}{|c|}{ SAC-CI } \\
\hline \multirow[b]{2}{*}{ State } & \multirow[b]{2}{*}{ Main configurations $(|C|>0.3)$} & \multirow[b]{2}{*}{ Nature } & \multirow{2}{*}{$\begin{array}{c}\text { Excitation } \\
\text { energy } \\
(\mathrm{eV})\end{array}$} & \multirow{2}{*}{$\begin{array}{l}\text { Oscillator } \\
\text { strength }\end{array}$} & \multicolumn{2}{|c|}{ Mulliken charge } & \multicolumn{2}{|c|}{$\begin{array}{c}\text { Dipole } \\
\text { moment }(\mathrm{D})^{\mathrm{a}}\end{array}$} & \multirow{2}{*}{$\begin{array}{c}\mathrm{CT} \\
\text { character }^{\mathrm{b}}\end{array}$} \\
\hline & & & & & $\mathrm{C}_{6} \mathrm{H}_{5}$ & $\mathrm{NH}_{2}$ & $\mu_{y}$ & $|\boldsymbol{\mu}|$ & \\
\hline$X^{1} A^{\prime}$ & (ground state) & $\cdots$ & 0.00 & $\cdots$ & +0.10 & -0.10 & -0.10 & 1.37 & $\cdots$ \\
\hline $2{ }^{1} A^{\prime}$ & $0.69(25-30)+0.59(24-31)$ & $\pi-\pi^{*}$ & 4.73 & 0.001 & +0.10 & -0.10 & -0.13 & 1.37 & LE \\
\hline $4{ }^{1} A^{\prime}$ & $0.65(24-30)-0.64(25-31)$ & $\pi-\pi^{*}$ & 6.19 & 0.008 & +0.13 & -0.13 & -0.38 & 1.32 & LE \\
\hline $2{ }^{1} A^{\prime \prime}$ & $0.64(23-31)-0.53(23-27)-0.44(23-30)$ & $n-\pi^{*}$ & 6.60 & 0.000 & -0.31 & +0.31 & 5.82 & 5.85 & $\mathrm{CT}$ \\
\hline $4{ }^{1} A^{\prime \prime}$ & $0.80(23-30)+0.47(23-31)$ & $n-\pi^{*}$ & 6.99 & 0.001 & -0.46 & +0.46 & 8.03 & 8.13 & $\mathrm{CT}$ \\
\hline $5{ }^{1} A^{\prime}$ & $0.62(24-27)+0.47(25-30)-0.41(24-31)+0.31(25-31)$ & $\pi$-diff.N $\left(p_{z}\right), \pi-\pi^{*}$ & 7.16 & 0.580 & +0.40 & -0.40 & -3.91 & 4.09 & $\mathrm{BCT}+\mathrm{LE}$ \\
\hline $6{ }^{1} A^{\prime}$ & $0.71(25-27)-0.54(24-30)$ & $\pi$-diff.N $\left(p_{z}\right), \pi-\pi^{*}$ & 7.26 & 0.487 & +0.52 & -0.52 & -6.10 & 6.23 & $\mathrm{BCT}+\mathrm{LE}$ \\
\hline $7^{1} A^{\prime}$ & $0.57(25-27)+0.56(25-31)+0.34(24-30)$ & $\pi$-diff.N $\left(p_{z}\right), \pi-\pi^{*}$ & 7.42 & 0.447 & +0.53 & -0.53 & -5.82 & 6.07 & $\mathrm{BCT}+\mathrm{LE}$ \\
\hline $8{ }^{1} A^{\prime}$ & $0.68(24-27)+0.48(24-31)-0.27(25-30)$ & $\pi$-diff.N $\left(p_{z}\right), \pi-\pi^{*}$ & 7.83 & 0.139 & +0.68 & -0.68 & -8.34 & 8.52 & $\mathrm{BCT}+\mathrm{LE}$ \\
\hline
\end{tabular}

${ }^{\mathrm{a}} \mu_{y}$ and $|\boldsymbol{\mu}|$ are the $y$-axis component and absolute value of the dipole moment, respectively.

${ }^{b} \mathrm{LE}=$ local excitation within the benzene ring, $\mathrm{CT}=$ charge transfer $\left(\mathrm{NH}_{2} \rightarrow \mathrm{C}_{6} \mathrm{H}_{5}\right), \mathrm{BCT}=$ back-CT $\left(\mathrm{C}_{6} \mathrm{H}_{5} \rightarrow \mathrm{NH}_{2}\right)$. 
TABLE VII. Energies and natures of ionized states of aniline.

\begin{tabular}{|c|c|c|c|c|c|c|c|c|c|}
\hline \multicolumn{5}{|c|}{ SAC-CI } & \multirow{2}{*}{$\begin{array}{l}\text { Koopmans } \\
\text { ionization } \\
\text { energy }(\mathrm{eV})\end{array}$} & \multicolumn{3}{|c|}{ Expt. (eV) } & \multirow{2}{*}{$\begin{array}{l}\text { Other theor. } \\
\frac{(\mathrm{eV})}{\mathrm{OVGF} /} \\
6-311 \mathrm{G}^{* * \mathrm{e}}\end{array}$} \\
\hline State & $\begin{array}{l}\text { Main configurations } \\
\qquad(|C|>0.3)\end{array}$ & Nature $^{a}$ & $\begin{array}{l}\text { Ionization } \\
\text { energy }(\mathrm{eV})\end{array}$ & Intensity $^{\mathrm{b}}$ & & $\begin{array}{l}\text { Kimura } \\
\text { et al. }{ }^{\mathrm{c}}\end{array}$ & $\begin{array}{l}\text { Palmer } \\
\text { et } a l^{\mathrm{d}}\end{array}$ & $\begin{array}{l}\text { Kishimoto } \\
\text { et al. }{ }^{\text {e }}\end{array}$ & \\
\hline $1^{2} A^{\prime}$ & $0.96(25)$ & $\pi^{+}$ & 7.66 & 0.939 & 8.07 & 8.00 & 8.05 & 8.05 & 7.68 \\
\hline $1^{2} A^{\prime \prime}$ & $0.97(24)$ & $\pi^{-}$ & 8.99 & 0.935 & 9.13 & 9.21 & 9.23 & 9.25 & 8.89 \\
\hline $2^{2} A^{\prime}$ & $0.94(23)$ & $\pi^{+}$ & 10.80 & 0.906 & 12.18 & 10.80 & 10.84 & 10.73 & 10.73 \\
\hline $2{ }^{2} A^{\prime \prime}$ & $0.96(22)$ & $\sigma^{-}$ & 11.78 & 0.930 & 13.26 & 11.74 & 11.82 & $11.7^{\mathrm{f}}$ & 11.82 \\
\hline $3^{2} A^{\prime}$ & $0.96(21)$ & $\sigma^{+}$ & 12.09 & 0.918 & 13.72 & 12.39 & 12.47 & $11.7^{\mathrm{f}}$ & 12.21 \\
\hline $4{ }^{2} A^{\prime}$ & $0.93(20)$ & $\pi^{+}$ & 12.76 & 0.873 & 14.48 & $\sim 12.6^{\mathrm{f}}$ & 12.47 & 12.3 & 12.77 \\
\hline $3^{2} A^{\prime \prime}$ & $0.89(19)+0.34(18)$ & $\sigma^{-}$ & 14.02 & 0.914 & 15.74 & 14.04 & 14.17 & 13.8 & 14.11 \\
\hline $4{ }^{2} A^{\prime \prime}$ & $0.88(18)-0.34(19)$ & $\sigma^{-}$ & 14.19 & 0.909 & 16.05 & 14.04 & 14.17 & 14.0 & 14.26 \\
\hline $5^{2} A^{\prime}$ & $0.95(17)$ & $\sigma^{+}$ & 14.36 & 0.909 & 16.19 & 14.04 & 14.17 & 14.3 & 14.52 \\
\hline $6^{2} A^{\prime}$ & $0.92(16)$ & $\sigma^{+}$ & 15.73 & 0.852 & 17.71 & 15.52 & 15.62 & 15.49 & 15.96 \\
\hline $5^{2} A^{\prime \prime}$ & $0.94(15)$ & $\sigma^{-}$ & 16.74 & 0.915 & 18.44 & $15.9^{\mathrm{f}}$ & & $15.9^{\mathrm{f}}$ & 16.95 \\
\hline $7^{2} A^{\prime}$ & $0.94(14)$ & $\sigma^{+}$ & 17.00 & 0.880 & 19.05 & 16.77 & 16.84 & 16.80 & 17.15 \\
\hline $8^{2} A^{\prime}$ & $0.91(13)$ & $\sigma^{+}$ & 19.92 & 0.830 & 22.01 & & 18.9 & & \\
\hline $6^{2} A^{\prime \prime}$ & $0.77(12)$ & $\sigma^{-}$ & 19.95 & 0.590 & 22.56 & & 19.2 & & \\
\hline
\end{tabular}

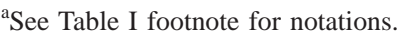

${ }^{\mathrm{b}}$ Estimated using monopole approximation.

${ }^{\mathrm{c}}$ Reference 11.

${ }^{\mathrm{d}}$ Reference 10 .

${ }^{\mathrm{e}}$ Reference 12 .

${ }^{\mathrm{f}}$ Values in italic are those of shoulder peaks.

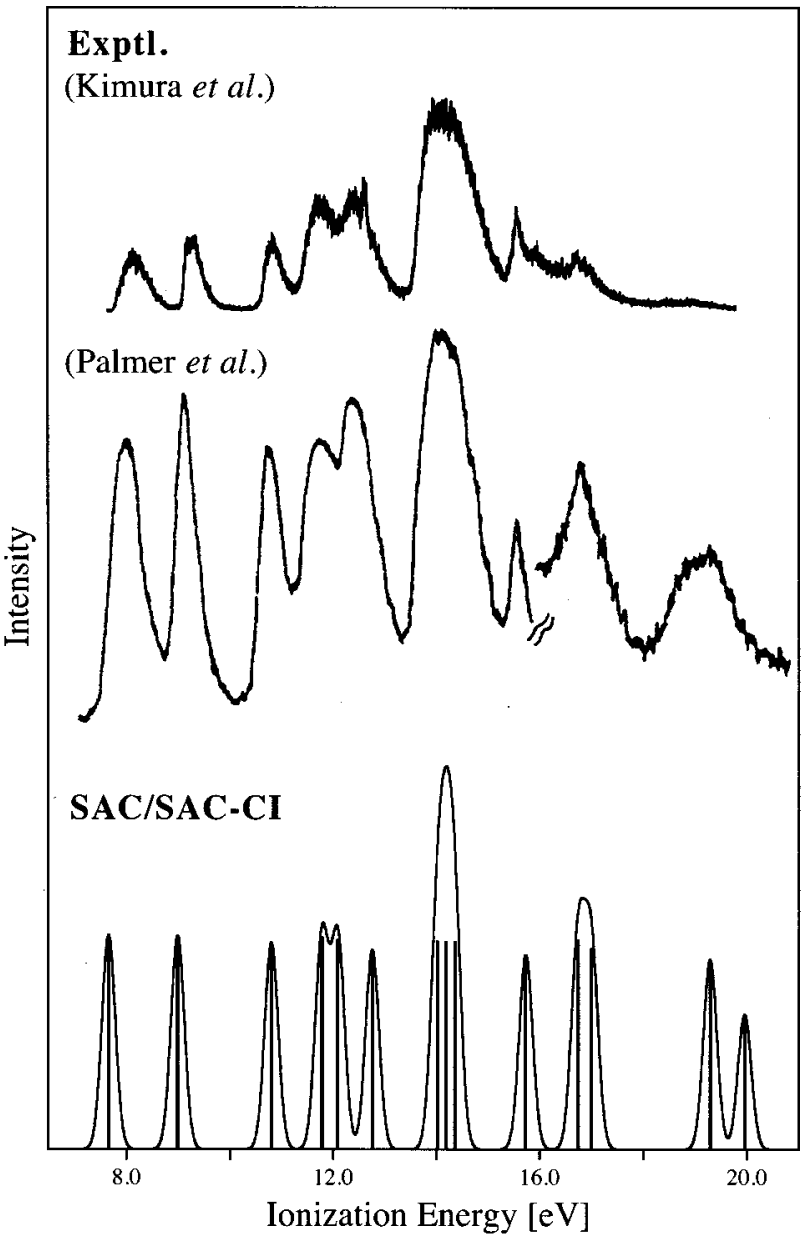

FIG. 5. Ionization spectrum calculated by SAC-CI with those observed by Kimura et al. (Ref. 11) and Palmer et al. (Ref. 10). sets may give an incorrect order. Assignments in Ref. 11 were based on the HF/4-31G calculation. Those assignments differ from ours at higher ionized states. Because the shake-up processes are unimportant, the OVGF calculations by Kishimoto et al. ${ }^{12}$ also provided the same order as ours. The lowest three states $\left(1^{2} A^{\prime}, 1^{2} A^{\prime \prime}\right.$, and $\left.2^{2} A^{\prime}\right)$ are assigned to ionizations from $\pi$ orbitals, although the third one also has an ionization character from the $n$ orbital. The sixth state $\left(4^{2} A^{\prime}\right)$ is also assigned to a $\pi$ ionization. Other states are due to $\sigma$ ionizations. In summary, ionized states are assigned to $\pi^{+}, \pi^{-}, \pi^{+}, \sigma^{-}, \sigma^{+}, \pi^{+}, \sigma^{-}, \sigma^{-}, \sigma^{+}, \sigma^{+}$, $\sigma^{-}, \sigma^{+}, \sigma^{+}, \sigma^{-}$in increasing-energy order. They correspond to ${ }^{2} B_{1},{ }^{2} A_{2},{ }^{2} B_{1},{ }^{2} B_{2},{ }^{2} A_{1},{ }^{2} B_{1},{ }^{2} B_{2},{ }^{2} B_{2}$, ${ }^{2} A_{1},{ }^{2} A_{1},{ }^{2} B_{2},{ }^{2} A_{1},{ }^{2} A_{1}$, and ${ }^{2} B_{2}$ for $C_{2 v}$ symmetry.

Ionization bands observed at 22.1 and $23.0 \mathrm{eV}$ by Palmer et al. ${ }^{10}$ are expected to contain shake-up processes: for reliable study on the shake-up peaks, it is necessary to employ the SAC-CI general- $R$ method. ${ }^{17,41}$

\section{SUMMARY}

We studied ground, excited, and ionized states of aniline using the SAC/SAC-CI method. Calculated singlet excitation energies showed good agreement with the experimental values. Absorption bands are assigned to ${ }^{1} A^{\prime \prime}\left(\sim^{1} B_{2}\right),{ }^{1} A^{\prime}$ $\left(\sim^{1} A_{1}\right),{ }^{1} A^{\prime \prime} \quad\left(\sim^{1} B_{2}\right), \quad{ }^{1} A^{\prime} \quad\left(\sim^{1} A_{1}\right),{ }^{1} A^{\prime \prime} \quad\left(\sim^{1} B_{2}\right)$ in increasing-energy order having mainly $\pi-\pi^{*}$ natures. An $s$-Rydberg state was found to lie between first and second $\pi-\pi^{*}$ states, supporting recent experimental results of Ebata et $a l .{ }^{37}$ The lowest band has a charge-resonance (CR) character with a slight charge-transfer (CT) nature. The third and fifth valence bands have a back-CT (BCT) nature, while the second and fourth have local excitation (LE) nature. The 
CT characters of excited states are dependent upon conformation of the amino group. In particular, the spectra and natures of excited states were largely altered in the twisted form; third and fourth lowest valence states exhibited strong CT characters while the fifth to eighth states showed strong BCT natures. This implies that aniline can have CT and BCT excited states if the amino group is twisted.

The ionization spectrum calculated by the SAC-CI method agreed well with experimental ones. Ionization bands are assigned to be $\pi^{+}, \pi^{-}, \pi^{+}, \sigma^{-}, \sigma^{+}, \pi^{+}, \sigma^{-}$, $\sigma^{-}, \sigma^{+}, \sigma^{+}, \sigma^{-}, \sigma^{+}, \sigma^{+}$, and $\sigma^{-}$in increasing-energy order. Koopmans theorem gives identical order, but the values are rather poor. These bands are due mainly to oneelectron ionization; effects of the "shake-up" process seem to be small.

\section{ACKNOWLEDGMENTS}

We are grateful to Ms. Minejima, Dr. Ebata, and Professor Mikami in Tohoku University for valuable discussions. Part of this study has been supported by a Grant-in-Aid for Scientific Research from the Japanese Ministry of Education, Science, and Culture. Y.H. acknowledges a fellowship from the Japan Society for the Promotion of Science (JSPS).

${ }^{1}$ K. Kimura, H. Tsubomura, and S. Nagakura, Bull. Chem. Soc. Jpn. 37, 1336 (1964).

${ }^{2}$ K. Kimura and S. Nagakura, Mol. Phys. 9, 117 (1965).

${ }^{3}$ J. N. Murrell, Proc. Phys. Soc., London, Sect. A 68, 969 (1955).

${ }^{4}$ H. Baba, Bull. Chem. Soc. Jpn. 34, 76 (1961).

${ }^{5}$ I. Fischer-Hjalmars, Ark. Fys. 21, 123 (1962).

${ }^{6}$ S. Suzuki and T. Fujii, Bull. Chem. Soc. Jpn. 48, 835 (1975).

${ }^{7}$ F. Bertinelli, A. Brillante, P. Palmieri, and C. Taliani, Gazz. Chim. Ital. 110, 321 (1980).

${ }^{8}$ J. M. Leal and B. Garcia, Z. Phys. Chem. (Leipzig) 269, 26 (1988).

${ }^{9}$ K. Fuke and S. Nagakura, J. Mol. Spectrosc. 64, 139 (1977).

${ }^{10}$ M. H. Palmer, W. Moyes, M. Spiers, and J. N. A. Ridyard, J. Mol. Struct. 53, 235 (1979).

${ }^{11}$ K. Kimura, S. Katsumata, Y. Achiba, T. Yamazaki, and S. Iwata, Handbook of HeI Photoelectron Spectra of Fundamental Organic Molecules (Japan Scientific Societies, Tokyo, 1981), p. 190.

${ }^{12}$ N. Kishimoto, M. Furuhashi, and K. Ohno, J. Electron Spectrosc. Relat. Phenom. 113, 35 (2000).
${ }^{13}$ H. Nakatsuji and K. Hirao, J. Chem. Phys. 68, 2053 (1978).

${ }^{14}$ H. Nakatsuji, Chem. Phys. Lett. 59, 362 (1978).

${ }^{15}$ H. Nakatsuji, Chem. Phys. Lett. 67, 329 (1979); 67, 334 (1979).

${ }^{16}$ H. Nakatsuji, Acta Chim. Hung. 129, 719 (1992).

${ }^{17} \mathrm{H}$. Nakatsuji, Computational Chemistry, Reviews of Current Trends, edited by J. Leszczynski (World Scientific, Singapore, 1997), Vol. 2.

${ }^{18}$ See, e.g., A. Broo and M. C. Zerner, Chem. Phys. Lett. 227, 551 (1994); U. Lommatzsch and B. Brutschy, Chem. Phys. 234, 35 (1998); A. B. J. Parusel, G. Köhler, and S. Grimme, J. Phys. Chem. A 102, 6297 (1998), and references are therein.

${ }^{19}$ T. H. Dunning Jr., J. Chem. Phys. 53, 2823 (1970).

${ }^{20} \mathrm{~S}$. Huzinaga, J. Andzelm, M. Klobukowski, E. Radzio-Andzelm, Y. Sakai, and H. Tatewaki, Gaussian Basis Set for Molecular Calculations (Elsevier, Amsterdam, 1984).

${ }^{21}$ T. H. Dunning Jr. and P. J. Hay, Modern Theoretical Chemistry, edited by H. F. Schaefer III (Plenum, New York, 1977), Vol. 3, p. 1.

${ }^{22}$ G. Schultz, G. Portalone, F. Ramondo, A. Domenicano, and I. Hargittai, Struct. Chem. 7, 59 (1996).

${ }^{23}$ H. Nakatsuji, Chem. Phys. 75, 425 (1983).

${ }^{24}$ H. Nakatsuji, J. Hasegawa, and M. Hada, J. Chem. Phys. 104, 2321 (1996).

${ }^{25}$ Y. Tokita, J. Hasegawa, and H. Nakatsuji, J. Phys. Chem. A 102, 1843 (1998).

${ }^{26}$ H. Nakatsuji, M. Hada, M. Ehara, J. Hasegawa, T. Nakajima, H. Nakai, O. Kitao, and K. Toyota, SAC/SAC-CI program system (SAC-CI 96) for calculating ground, excited, ionized, and electron attached states and singlet to septet spin multiplicities (Kyoto University, Kyoto, 1998).

${ }^{27}$ M. J. Frisch, G. W. Trucks, H. B. Schlegel et al., GAussian 98, Revision A.1, Gaussian Inc., Pittsburgh, PA, 1998.

${ }^{28}$ D. G. Lister, J. K. Tyler, J. H. Høg, and N. W. Larsen, J. Mol. Struct. 23, 253 (1974).

${ }^{29}$ A. L. McClellan, Tables of Experimental Dipole Moments (Rahara Enterprises, El Cerrito, 1974), Vol. II.

${ }^{30}$ J. R. Lombardi, J. Chem. Phys. 50, 3780 (1969).

${ }^{31}$ J. R. Lombardi, J. Chem. Phys. 56, 2278 (1972).

${ }^{32}$ J. R. Lombardi, Chem. Phys. 28, 41 (1978).

${ }^{33}$ T. M. Korter, D. R. Borst, C. J. Butler, and D. W. Pratt, J. Am. Chem. Soc. 123, 96 (2001).

${ }^{34}$ H. Tsubomura and T. Sakata, Chem. Phys. Lett. 21, 511 (1973).

${ }^{35}$ A. Davidson and B. Nordén, Chem. Phys. Lett. 28, 39 (1974).

${ }^{36}$ M. B. Robin, Higher Excited States of Polyatomic Molecules (Academic, New York, 1975), Vol. II, p. 255.

${ }^{37}$ T. Ebata, C. Minejima, and N. Mikami, J. Chem. Phys. (submitted).

${ }^{38}$ A. Hiraya and K. Shobatake, J. Chem. Phys. 94, 7700 (1991).

${ }^{39}$ E. N. Lassetre, A. Skerbele, M. A. Dilon, and K. J. Ross, J. Chem. Phys. 48, 5066 (1968)

${ }^{40}$ R. L. Martin and D. A. Shirley, J. Chem. Phys. 64, 3685 (1976).

${ }^{41}$ H. Nakatsuji, Chem. Phys. Lett. 177, 331 (1991). 